\title{
DIRECT CLASS IV RESTORATION: TECHNICAL REPORT
}

\author{
Dr. Idiberto Jose Zotarelli Filho, MSc, Ph.D ${ }^{1}$ \\ ${ }^{1}$ Affiliation not available
}

June 8, 2020

\begin{abstract}
The objective of this work was to approach a freehand restorative technique for class IV in composite resin. The procedure was performed on a mannequin containing elements $12,11,21$ and 22 , the technique was performed on element 21 , the same was provided with the ready preparation, vestibular bevel, drill used with 1190 tapered diamond tip, the resins of choice were CT, dentin A3, body A3, WB and enamel A2 all from Z350 - 3M, as each contains different chromas. Today for restorations on teeth older than $3 \mathrm{M}$ it is the best option on the market, with the patent for nanoparticulate resins. The professional must learn the rules of aesthetics of natural teeth for the use of these materials. Treating that natural teeth are polychromatic, while composite resins are monochromatic. Given the existence of a wide variety of resins and technical possibilities, the following text proposes a clinical sequence of reconstruction of anterior teeth with compromised incisal angle due to fracture or caries. Restorative rehabilitation, in addition to promoting health, provides a favorable aesthetic appearance for the smile of the patient, thus raising the patient's self-esteem. The introduction of the acid conditioning technique and the development of adhesive systems associated with improvements in the mechanical properties of composite resins have provided new perspectives for aesthetic dentistry.
\end{abstract}

Keywords: Restoration. Composite resin. Anterior teeth. Class IV anterior teeth. Direct restorative technique.

\section{Maria Eduarda Barbosa Cardoso ${ }^{1}$, Yan Lomba Ronchi ${ }^{1}$, Idiberto José Zotarelli Filho ${ }^{1,2,3}$, Taylane Soffener Berlanga de Araújo ${ }^{1,2}$}

\author{
1 University Center North Paulista (Unorp), Graduate in Dentistry, São José do Rio Preto - \\ SP, Brazil.
}

2 Post graduate and continuing education (Unipos), Postgraduate in dentistry, São José do Rio Preto SP, Brazil.

\section{Bentham Science Ambassador, Brazil.}

\section{Introduction}

For a long time, most of the destruction of the incisal angle was due to lesions of interproximal caries. Nowadays, these losses occur mainly due to injuries. ${ }^{1}$ They are more frequent in the upper anterior teeth of male adolescents. ${ }^{2}$

In the unavailability or impracticability of using the dental fragment, bonding is no longer an alternative treatment, where we will have the use of adhesive systems and composite resins for direct use. ${ }^{3}$ The growing technological innovation, current composite resins, especially nanoparticles, manage to gather functional 
mechanical characteristics for regions subjected to high stresses, with adequate optical properties to achieve aesthetic excellence in restoring anterior teeth. ${ }^{4,5}$

The longevity of restorations in anterior teeth depends on the preparation of the cavity, material used and technique developed. ${ }^{6}$ When the incisal angle is compromised in canines and incisors, there is a reduction in the average survival time of the restoration. ${ }^{7}$

The objective of this work was to approach the direct hand restorative technique of class IV anterior teeth, emphasizing the clinical protocols, materials used, step by step of the restoration execution.

\section{Methods}

\section{Study Design}

Meta-analysis, case reports, retrospective, prospective and randomized studies with qualitative and/or quantitative analysis were included. Initially, keywords were determined by searching the DeCS tool (Descriptors in Health Sciences, BIREME base) and then verified and validated by the MeSH System (Medical Subject Headings, the US National Library of Medicine) to achieve a consistent search.

The present study was about a description and technical report on direct restoration of anterior teeth followed by a literary review. The main descriptors (Mesh Terms) used were "Restoration. Composite resin. Anterior teeth. Class IV anterior teeth. Direct restorative technique". For further specifications, the description "Direct restorative technique" for refinement was added during the research, following the rules of CARE - Case Reports Guidelines. Available at: https:/www.care-statement.org/). The bibliographic search was carried out through online databases: PUBMED, SCOPUS, COCHRANE LIBRARY AND GOOGLE SCHOLAR. The deadline and related research were set, covering all available literature on virtual libraries.

\section{Series of Articles and Eligibility For Literary Review}

A total of 55 articles were found involving implantology and bisphosphonate. Initially, the existing title was excluded and duplicated according to the interest described in this study. After this process, the abstracts were evaluated and a new exclusion was performed. A total of 21 articles were evaluated in full and 15 were included and discussed in this study.

\section{Technical Report}

For direct adhesive restorations on fractured anterior teeth, it is important to note the following factors: anamnesis, trauma etiology, assessment of oral health status and extent of the fracture, radiographic examination, degree of periodontal involvement and pulp.

In cases of caries lesion, the proximal face presents favorable conditions for the retention of plaque and the beginning of the development of the carious lesion, progressing through the enamel prisms and dentinal tubules, deeply reaching the enamel and dentin. ${ }^{10}$

\section{Isolation of the operative field}

Adhesive techniques require an operative field without contamination and moisture. It is recommended to use absolute isolation with a canine-to-canine rubber dam with clamps on premolars or clamp $\mathrm{n}^{\mathrm{O}} 212$ on the tooth to be restored stabilized with godiva. ${ }^{4}$ On the mannequin absolute isolation was made with strings with dental floss on all upper incisors.

\section{Tooth preparation}

After cleaning the surface with a pumice stone to remove any extrinsic stains, the colors necessary for the restoration were determined before preparing the tooth or placing the insulation. A dry tooth will be lighter than a healthy tooth. ${ }^{4}$

Regarding the preparation of the tooth, in addition to the removal of decayed tissue if present, there are basically two alternatives: The non-execution of any type of preparation and the preparation of a bevel 
preparation with one. Its advantages are the defined marginal finish, an adequate adaptation of the resin, less risk of excess resin. ${ }^{4}$

A Fillon thread sealing tape is placed on neighboring teeth to protect them from acid and adhesive attacks by not allowing them to touch adjacent teeth. 37\% phosphoric acid was applied over the enamel for 15 seconds. After that, the acid was washed with abundant water and dried with gentle air jets according to the manufacturer's instructions.

With a disposable micro-applicator, the adhesive system is applied, using the Single Bond Universal 3M adhesive for 10 seconds, air jets and 20 seconds of polymerization. The freehand technique was performed, where a polyester tape was placed on the palatal face of the tooth for the insertion of Filtek Z350 XT CT Translucent - 3M resin to make a thin palatal wall. The photopolymerized must be positioned so that it gently touches the buccal and palatal surfaces, respectively, being activated for 20 seconds according to the manufacturer. In the incisal space, space was left to insert a translucent resin.

To avoid pre-polymerization of the resin, the reflector light must be removed from the working area. The increment of the resin cannot exceed $2 \mathrm{~mm}$ of thickness. ${ }^{9}$ Afterwards, with a spatula of insertion, the increment of resin Filtek Z350 A3 Dentin is placed to make the artificial dentine, being polymerized for 20 seconds. Then, the Filtek Z350 A3 Corpo resin was used on the dentin resin, polymerized for 20 seconds. Throughout the incisal third, with the help of a brush, an increment of Filtek Z350 WB resin was placed to make the incisal halo and polymerized for 30 seconds. After that, the last layer of Filtek Z350 A2 Enamel resin was added, this was positioned with an insertion spatula and sculpted with the help of a brush.

\section{Functional fit}

The functional adjustment must precede the finishing and polishing. ${ }^{4}$ For this, it is necessary before the restoration to check the contact points in maximum habitual intercuspation (MIH), using a carbon (AccuFilm). Check-in which teeth the lateral and protrusive disocclusion guides occur and try not to modify them.

After the restoration, check the MIH contacts again, and if there is any unwanted contact, reduce it with a fine-grained diamond tip so that the points coincide with those marked before the restoration. ${ }^{6}$

\section{Finishing and polishing}

The finishing can be done with multi-laminated drills or fine-grained and extra-fine diamond tips in high rotation. Spherical tips and pear-shaped or barrel tips can also be used in order to remove the coarsest excesses of the material.4 The finishing of the proximal surfaces can be done with the help of sanding strips $2 \mathrm{~mm}$ wide. ${ }^{4}$

The ideal polishing should be done after seven days, in the nanohybrid composites the restorations were smoother making the polishing immediately but without irrigation10. Felt discs with diamond paste and flexible sequential discs (Sof-Lex Pop On 3M Sanding Disc) were used.

\section{Discussion}

The resins presented in this work were Filtek Z350 3M. This was the first product that used nanotechnology and is the only one on the market to offer the aesthetics of a microparticulate resin and the resistance of a hybrid resin, according to the manufacturer. Selection involves determining the hue, value, and chroma. The selection must be made based on factors related to the material itself, on clinical factors, on the professional's preference. ${ }^{1,2}$

Natural teeth are polychromatic, and while composite resins are monochromatic, it is therefore very difficult to achieve aesthetic excellence with a single color of composite resin, although they do not always compromise the smile's aesthetic. ${ }^{3,4}$ Inadequate light curing can lead to undesirable consequences such as: decreased adhesion of adhesives, decreased color stability of the composite resin due to a partial reaction of 
the accelerator and absorption of dyes, decreased wear resistance due to inadequate mechanical property of this material. ${ }^{4}$

Thus, direct restorations of the anterior teeth, especially in the central incisors, symmetry plays an important role. The clinician can take advantage of silicone indices based on a wax to build palate and incisal walls; however, when he needs to reproduce symmetrical items on the side of the chair freehand, such as interproximal emergency profiles, macro, and micro-surface textures and chromatic characteristics, the result can often be unpredictable. ${ }^{11}$

In this context, a meta-analysis study discussed the effectiveness of composite resin restorations on anterior teeth, verifying whether specific material classes, dental conditioning methods, and operational procedures influence the outcome for Class III and Class IV restorations. Of the 84 clinical trials, 21 studies met the inclusion criteria, 14 of them for Class III restorations, 6 for Class IV restorations, and 1 for diastema closure; the latter was included in the class IV group. Twelve of the 21 studies started before 1991 and 18 before 2001. The average estimated success rate (without replacement) after 10 years for Class III composite resin restorations was $95 \%$ and for Class IV restorations $90 \%$. The main reason for replacing Class IV restorations was mass fractures, which occurred significantly more frequently with macrofiliated composites than with hybrid and macrofiliated composites. Caries adjacent to restorations were uncommon in most studies and represented only $2.5 \%$ of all restorations replaced after 10 years, regardless of cavity class. Class III restorations with glass ionomer derivatives suffered significantly more anatomically loss than restorations with other types of material. When the enamel was etched with acid and no bonding agent was applied, significantly more restorations showed marginal staining and detectable margins compared to the enamel attack with enamel bonding or the total etching technique. The chamfer of the enamel was associated with a significantly reduced deterioration of the anatomical shape compared to non-chamfering, but not with less marginal spots or less detectable margins. The type of isolation (absolute / relative) had a statistically significant influence on marginal caries, which, however, can be a random finding. ${ }^{12}$

A 15-year retrospective study investigated the impact on survival, when a repair is seen as a failure or not, in composite anterior restorations. Data were collected from the files of patients at a private dental clinic, including patients with direct composite restorations placed on the anterior teeth (Class III, Class IV, or veneer) between January 1994 and December 2009. The data were analyzed considering whether or not to repair as a failure. One hundred and forty-four patient records were included, with 634 restorations. At 15 years of age, Class III / IV restorations showed $69 \%$ survival and $2.4 \%$ annual failure rate (AFR) when the repair was not considered a failure and $64 \%$ and $2.9 \%$ AFR, respectively when the repair was seen as a failure. For direct veneers, at 5 and 10 years of follow-up, survival dropped from $85 \%$ to $74 \%$ and from $52 \%$ to $38 \%$, respectively, when the repair was considered a failure. In general, restorations placed in the upper jaw had an increased risk of failure compared to the lower jaw, and restorations in central incisors had a higher risk of failure compared to dogs. Therefore, composite repair appears to be a suitable alternative for class III, class IV and veneer restorations, as it was able to increase the survival of restorations performed on anterior teeth. ${ }^{13}$

Also, a prospective observational cohort study aimed to estimate the survival of a hybrid compound placed by a clinician up to 8 years of follow-up. All patients were referred and recruited for a prospective observational

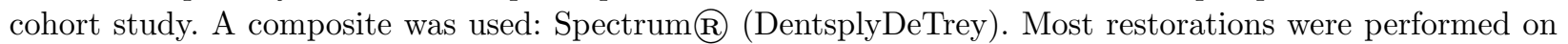
the upper anterior teeth using a Dahl approach. A total of 1010 direct composites were placed in 164 patients. The average follow-up time was 33.8 months. 71 of 1010 restorations failed during follow-up. The time to failure was significantly longer in older individuals and when there was a lack of posterior support. Bruxism and an increase in the vertical occlusal dimension were not associated with the failure. The proportion of failures was higher in patients with a Class 3 or edge-to-edge incisal relationship than in Class 1 and Class 2 cases, but this was not statistically significant. There were more failures in the lower arch (9.6\%) compared to the upper arch $(6 \%)$, with the largest number of composites being placed on the upper incisors $(\mathrm{n}=$ $519) \cdot{ }^{14}$

In this sense, creating perfect direct composite restorations is a challenge due to the limitations of many 
materials that affect the integration of shadow or surface quality and color stability. Along with technological disadvantages, a certain complexity and lack of predictability in the clinical application were inherent to the technique and made it elitist for a long time. The concepts of shading and layers have progressively evolved from a simplistic, known-histologic bilaminar technique to a multi-layer approach (3 to 4 or more layers), following the Vita Classic system. One of the most achieved concepts is polychromatic stratification, which uses a variable number of layers (basically VITA opaque dentin or not VITA, chromatic enamel, and translucent/opalescent enamel), driven by the natural optical composition of the tooth. Also, a simplified non-VITA shading system was developed with a reduced number of layers, with a layer of dentin and enamel and effect shades, known as the concept of natural stratification, aiming at the same optimal and natural aesthetic integration. ${ }^{15}$

\section{Competing interests}

The authors no have competing interests.

\section{Acknowledgement}

We appreciate greatly the UNIPOS graduate for support, also UNORP of Sao Jose do Rio Preto/SP;Brazil for the support.

\section{References}

1. Yanikian C, Yanikian F, Sundfeld D, Lins R, Martins L. Direct Composite Resin Veneers in Nonvital Teeth: A Still Viable Alternative to Mask Dark Substrates. Oper Dent. 2019;44(4):E159-E166. doi:10.2341/18-220-T.

2. Brown KM, Gillespie G. Advancements in Composite Resin Material Enable Streamlined Direct Restoration Process. Compend Contin Educ Dent. 2019;40(suppl 2):2-6.

3. Andreasen, JO. : Challenges in clinical dental traumatology. Endo Dent Traumatol 1:45-55, 1985.

4. Baratieri, Luiz Narciso/ et al.: Odontologia restauradora.11:399, 2001.

5. Cortes MI, Marcenes W, Sheiham A: Prevalence and correlates f traumatic injuries to the permanent teeth of schoolchildren aged 9-14 years in Belo Horizonte, Brazil. Dent Traumatol 2001;17(1):22-6.

6. Calixtro LR, Clareijo V,Kasbbach W, Andradea MF: Harmonizacao do sorriso com resina composta direta. Dental Press Estet 2009;6(1):18-28.

7. Lucarotti PSK, Holder RL, Burke FJT: Outcome of direct restorations placed within the general dental services in England and Wales (Part I) variation by type of restoration and re-intervention. J Dent 2005;33(10):805-15.

8. Paz, Sofia Fonseca DA Costa: Diferentes sistemas de polimento das resinas compostas. Qual o melhor. Porto, 2018.

9. Stolf, Sheila Cristina: Fotopolimerizacao das resinas compostas. 3:18, 2004.

10. Van Dijken JW, Pallesen U: Fracture frenquency and longevity of fracturedresin composite, polyacidmodified resin composite, and resin modified glass ionomer cement class IV restorations: an um to 14 years of follow-up. Clin Oral Investig 2010;14(2):217-22.

11. Paolone G. Direct composite restorations in anterior teeth. Managing symmetry in central incisors. Int J Esthet Dent. 2014;9(1):12-25.

12. Heintze SD, Rousson V, Hickel R. Clinical effectiveness of direct anterior restorations-a metaanalysis. Dent Mater. 2015;31(5):481-495. doi:10.1016/j.dental.2015.01.015.

13. Van de Sande FH, Moraes RR, Elias RV, et al. Is composite repair suitable for anterior restorations? A long-term practice-based clinical study. Clin Oral Investig. 2019;23(6):2795-2803. doi:10.1007/s00784018-2722-5.

14. Milosevic A, Burnside G. The survival of direct composite restorations in the management of severe tooth wear including attrition and erosion: A prospective 8-year study. J Dent. 2016; 44:13-19. doi:10.1016/j.jdent.2015.10.015.

15. Dietschi D, Fahl N Jr. Shading concepts and layering techniques to master direct anterior composite restorations: an update. Br Dent J. 2016;221(12):765-771. doi:10.1038/sj.bdj.2016.944. 